\title{
Brain oscillations and predictive processing
}

\section{Juliana Yordanova*, Vasil Kolev and Roumen Kirov*}

Institute of Neurobiology, Bulgarian Academy of Sciences, Sofia, Bulgaria

*Correspondence: jyord@bio.bas.bg; ru@bio.bas.bg

Edited by:

Shimon Edelman, Cornell University, USA

Reviewed by:

Axel Cleeremans, Université Libre de Bruxelles, Belgium

\section{A commentary on}

Whatever next? Predictive brains, situated agents, and the future of cognitive science by Clark, A. (in press). Behav. Brain Sci.

The hierarchical generative model presented in the target article is based on theoretical and computational grounds. However, providing neurophysiologic evidence for the presence of distinct neural mechanisms mediating top-down predictions and bottom-up transmission of prediction error is critical for model validity.

In human cognitive electrophysiology, several event-related potentials (ERPs) are related to the mechanisms of comparing incoming with expected events. These are the mismatch negativity (MMN), errorrelated negativity (ERN), and feedbackrelated negativity (FRN) operating basically in the sensory, action/performance, and cognitive domains, respectively. These ERP components are all correlated with generating and transmitting mismatch information (Falkenstein et al., 1991; Nieuwenhuis et al., 2004; Näätänen et al., 2007) and have been previously targeted as markers of bottomup prediction error (Garrido et al., 2009; Cavanagh et al., 2010; Friston, 2012). There are, however, several issues that may question the suitability of these components for testing the predictive coding model.

The latencies of mismatch ERP components are relatively long (150-250 ms for MMN, 200-300 ms for FRN, and around $100 \mathrm{~ms}$ for ERN) under the assumption that they reflect a feed-forward "error" input from lower processing levels to the cortex. More critically, there is experimental evidence that much earlier ERP components are sensitive to deviance. For example, brainstem and middle-latency components within 10-80 ms (Slabu et al., 2010, 2012), the P1 component within 10-100 ms (Bendixen et al., 2009), and early gamma
$(>30 \mathrm{~Hz})$ oscillatory responses at around $50 \mathrm{~ms}$ of auditory ERPs (Schadow et al., 2009) discriminate deviant from standard stimuli. Further, the sources of auditory MMN have been localized in the bilateral temporal and in the prefrontal cortices (Giard et al., 1990; Rinne et al., 2000), the latter supposed to mediate a switch/reallocation of attention focus (Escera et al., 1998, 2003). Likewise, the sources of ERN and FRN are localized in the medial frontal and prefrontal brain regions (Ullsperger and von Cramon, 2001; Holroyd et al., 2004; Cohen et al., 2011). These data show that the "error" negativities of human ERPs may not represent the virtual error information transmitted to the cortex from lower processing levels. Rather, they may reflect a follow-up utilization of the mismatch information that would be incorporated to update and optimize top-down representations (via attention, memory, and emotional activations). Thus, the feedforward "error" signal presumed by the predictive coding theory should be sought in much earlier time windows after stimulus and even before stimulus (Romei et al., 2010). Referring to the classical "error" or "prediction-violation" ERP components needs to be conceptually clarified.

In search for error prediction ERP signatures, it is important to recognize that ERP components have a complex heterogeneous structure composed of frequencyspecific oscillations (Kolev et al., 1997; Yordanova et al., 2000). The ERN, in particular, comprises at least two oscillatory sub-components that carry error signals from co-existent processing systems (theta $-4-7 \mathrm{~Hz}$ and delta $-<4 \mathrm{~Hz}$ ) operating in parallel at the level of movement and behavioral control (Yordanova et al., 2004). It is remarkable that the oscillatory theta component has been detected not only for ERN (Luu and Tucker, 2001; Yordanova et al., 2004; Cavanagh et al., 2009, 2010), but it has also been established as a primary constituent of MMN (Fuentemilla et al., 2008; Hsiao et al., 2009; Ko et al., 2012) and FRN (Cohen et al., 2007, 2011; Marco-Pallares et al., 2008; Christie and Tata, 2009). Thus, the oscillatory theta activity may be a consistent neurophysiologic marker of the utilization of the mismatch (error) information by the executive-control systems in the cortex.

In contrast, there is evidence that increased fast-frequency (beta/gamma 15-80 Hz) oscillations may signal match detection. Cohen et al. (2007) and MarcoPallares et al. (2008) demonstrated that positive feedback (prediction confirmation) was associated with increased beta/ gamma activity, in contrast to negative feedback characterized by enhanced theta oscillations. Schadow et al. (2009) showed that early phase-locked gamma-band responses $(20-80 \mathrm{~Hz})$ were significantly increased for predictable regular auditory stimuli as compared to deviant stimuli, as found for fast-frequency responses in the brainstem (Slabu et al., 2012). This is in line with a model according to which matching between incoming stimulus and a memory template results in enhanced gamma-band activity (Herrmann et al., 2004). Thus, time-frequency decomposition of brain responses may be critical for identifying error signals in functional systems operating in parallel at different processing levels.

In the context of the generative predictive model, a major focus of exploration needs to be placed on error processing in different brain states, in which top-down executive-control mechanisms are reduced, totally inhibited, or qualitatively altered. Such states are best represented by different stages of sleep, anesthesia, coma, and brain diseases. For example, neuroimaging studies have demonstrated that subcortical regions and extrastriate visual cortices are more active during rapid eye movement (REM) sleep compared with wake and non-REM 
sleep, whereas executive-control (dorsolateral prefrontal and parietal) and primary visual cortices are suppressed in REM sleep compared with wake; In non-REM sleep, the activity of all brain areas relevant for information processing during wake is mostly suppressed (Maquet et al., 1996; Braun et al., 1997, 1998; Nofzinger et al., 1997). Interestingly, although inconsistent and contradictory, there is some evidence that in REM sleep, an analog of the MMN emerges despite the gross suppression of both executive top-down processing and external input transmission (Atienza et al., 2000; Ibáñez et al., 2009). No MMN has been reliably recorded during non-REM sleep and slow wave sleep (Ibáñez et al., 2009; Sculthorpe et al., 2009). It remains poorly understood if and how brain states differing dramatically in top-down and bottom-up mechanisms, neurochemistry, synaptic connectivity, and neuroelectric signaling such as sleep stages (Hobson et al., 2000; Hobson and Pace-Schott, 2002; Nir and Tononi, 2010) affect deviance detection, if inherent (free of executive-control prediction) error signals can be generated, and the extent to which the proposed dynamic Bayesian predictions are moderated by consciousness. Probing generative prediction theory would certainly benefit from referring to such models.

\section{REFERENCES}

Atienza, M., Cantero, J. L., and Gómez, C. M. (2000). Decay time of the auditory sensory memory trace during wakefulness and REM sleep. Psychophysiology 37, 485-493.

Bendixen, A., Schröger, E., and Winkler, I. (2009). I heard that coming: event-related potential evidence for stimulus-driven prediction in the auditory system. J. Neurosci. 29, 8447-8451.

Braun, A. R., Balkin, T. J., Wesensten, N. J., Gwadry, F., Carson, R. E., Varga, M., et al. (1998). Dissociated pattern of activity in visual cortices and their projections during human rapid eye movement sleep. Science 279, 91-95.

Braun, A. R., Balkin, T. J., Wesenten, N. J., Carson, R. E., Varga, M., Baldwin, P., et al. (1997). Regional cerebral blood flow throughout the sleep-wake cycle. An H2(15)O PET study. Brain 120, 1173-1197.

Cavanagh, J. F., Cohen, M. X., and Allen, J. J. (2009). Prelude to and resolution of an error: EEG phase synchrony reveals cognitive control dynamics during action monitoring. J. Neurosci. 29, 98-105.

Cavanagh, J. F., Frank, M. J., Klein, T. J., and Allen, J. J. (2010). Frontal theta links prediction errors to behavioral adaptation in reinforcement learning. Neuroimage 49, 3198-3209.

Christie, G. J., and Tata, M. S. (2009). Right frontal cortex generates reward-related theta-band oscillatory activity. Neuroimage 48, 415-422.
Cohen, M. X., Elger, C. E., and Ranganath, C. (2007). Reward expectation modulates feedback-related negativity and EEG spectra. Neuroimage 35, 968-978.

Cohen, M. X., Wilmes, K., and Vijver, I. V. (2011). Cortical electrophysiological network dynamics of feedback learning. Trends Cogn. Sci. (Regul. Ed.) 15, 558-566.

Escera, C., Alho, K., Winkler, I., and Näätänen, R. (1998). Neural mechanisms of involuntary attention to acoustic novelty and change. J. Cogn. Neurosci. 10, 590-604.

Escera, C., Yago, E., Corral, M. J., Corbera, S., and Nuñez, M.I. (2003).Attention capture by auditory significant stimuli: semantic analysis follows attention switching. Eur. J. Neurosci. 8, 2408-2412.

Falkenstein, M., Hohnsbein, J., Hoormann, J., and Blanke, L. (1991). Effects of crossmodal divided attention on late ERP components. II. Error processing in choice reaction tasks. Electroencephalogr. Clin. Neurophysiol. 78, 447-455.

Friston, K. (2012). Prediction, perception and agency. Int. J. Psychophysiol. 83, 248-252.

Fuentemilla, L., Marco-Pallarés, J., Münte, T. F., and Grau, C. (2008). Theta EEG oscillatory activity and auditory change detection. Brain Res. 1220, 93-101.

Garrido, M. I., Kilner, J. M., Kiebel, S. J., and Friston, K. J. (2009). Dynamic causal modeling of the response to frequency deviants. J. Neurophysiol. 101, 2620-2631.

Giard, M. H., Perrin, F., Pernier, J., and Bouchet, P. (1990). Brain generators implicated in the processing of auditory stimulus deviance: a topographic event-related potential study. Psychophysiology 27, 627-640.

Herrmann, C. S., Munk, M. H., and Engel, A. K. (2004) Cognitive functions of gamma-band activity: memory match and utilization. Trends Cogn. Sci. (Regul. Ed.) 8, 347-355.

Hobson, J.A., and Pace-Schott, E. F. (2002). The cognitive neuroscience of sleep: neuronal systems, consciousness and learning. Nat. Rev. Neurosci. 3, 679-693.

Hobson, J. A., Pace-Schott, E. F., and Stickgold, R. (2000). Dreaming and the brain: toward a cognitive neuroscience of conscious states. Behav. Brain Sci.23, 793-842; discussion 904-1121.

Holroyd, C. B., Nieuwenhuis, S., Yeung, N., Nystrom, L., Mars, R. B., Coles, M. G., et al. (2004). Dorsal anterior cingulate cortex shows fMRI response to internal and external error signals. Nat. Neurosci. 7, 497-498.

Hsiao, F. J., Wu, Z. A., Ho, L. T., and Lin, Y. Y. (2009). Theta oscillation during auditory change detection: an MEG study. Biol. Psychol. 81, 58-66.

Ibáñez, A. M., Martín, R. S., Hurtado, E., and López, V. (2009). ERPs studies of cognitive processing during sleep. Int. J. Psychol. 44, 290-304.

Ko, D., Kwon, S., Lee, G. T., Im, C. H., Kim, K. H., and Jung, K. Y. (2012). Theta oscillation related to the auditory discrimination process in mismatch negativity: oddball versus control paradigm. J. Clin. Neurol. 8, 35-42.

Kolev, V., Demiralp, T., Yordanova, J., Ademoglu, A. and Isoglu-Alkaç, U. (1997). Time-frequency analysis reveals multiple functional components during oddball P300. Neuroreport 8, 2061-2065.

Luu, P., and Tucker, D. M. (2001). Regulating action: alternating activation of midline frontal and motor cortical networks. Clin. Neurophysiol. 112, 1295-1306.

Maquet, P., Péters, J., Aerts, J., Delfiore, G., Degueldre, C., Luxen, A., et al. (1996). Functional neuroanatomy of human rapid-eye-movement sleep and dreaming. Nature 383, 163-166.

Marco-Pallares, J., Cucurell, D., Cunillera, T., García, R., Andrés-Pueyo, A., Münte, T. F., et al. (2008). Human oscillatory activity associated to reward processing in a gambling task. Neuropsychologia 46, 241-248.

Näätänen, R., Paavilainen, P., Rinne, T., and Alho, K. (2007). The mismatch negativity (MMN) in basic research of central auditory processing: a review. Clin. Neurophysiol. 118, 2544-2590.

Nieuwenhuis, S., Holroyd, C. B., Mol, N., and Coles, M. G. (2004). Reinforcement-related brain potentials from medial frontal cortex: origins and functional significance. Neurosci. Biobehav. Rev. 28, 441-448.

Nir, Y., and Tononi, G. (2010). Dreaming and the brain: from phenomenology to neurophysiology. Trends Cogn. Sci. (Regul. Ed.) 14, 88-100.

Nofzinger, E. A., Mintun, M. A., Wiseman, M., Kupfer, D. J., and Moore, R. Y. (1997). Forebrain activation in REM sleep: an FDG PET study. Brain Res. 770, 192-201.

Rinne, T., Alho, K., Ilmoniemi, R. J., Virtanen, J., and Näätänen, R. (2000). Separate time behaviors of the temporal and frontal mismatch negativity sources. Neuroimage 12, 14-19.

Romei, V., Gross, J., and Thut, G. (2010). On the role of prestimulus alpha rhythms over occipito-parietal areas in visual input regulation: correlation or causation? J. Neurosci. 30, 8692-8697.

Schadow, J., Lenz, D., Dettler, N., Fründ, I., and Herrmann, C. S. (2009). Early gamma-band responses reflect anticipatory top-down modulation in the auditory cortex. Neuroimage 47, 651-658.

Sculthorpe, L. D., Ouellet, D. R., and Campbell, K. B. (2009). MMN elicitation during natural sleep to violations of an auditory pattern. Brain Res. 1290, 52-62.

Slabu, L., Escera, C., Grimm, S., and Costa-Faidella, J. (2010). Early change detection in humans as revealed by auditory brainstem and middle-latency evoked potentials. Eur. J. Neurosci. 32, 859-865.

Slabu, L., Grimm, S., and Escera, C. (2012). Novelty detection in the human auditory brainstem. J. Neurosci. 32, 1447-1452.

Ullsperger, M., and von Cramon, D. Y. (2001). Subprocesses of performance monitoring: a dissociation of error processing and response competition revealed by event-related fMRI and ERPs. Neuroimage 14, 1387-1401.

Yordanova, J., Devrim, M., Kolev, V., Ademoglu, A., and Demiralp, T. (2000). Multiple time-frequency components account for the complex functional reactivity of P300. Neuroreport 11, 1097-1103.

Yordanova, J., Falkenstein, M., Hohnsbein, J., and Kolev, V. (2004). Parallel systems of error processing in the brain. Neuroimage 22, 590-602.

Received: 01 September 2012; accepted: 30 September 2012; published online: 17 October 2012.

Citation: Yordanova J, Kolev V and Kirov R (2012) Brain oscillations and predictive processing. Front. Psychology 3:416. doi: 10.3389/fpsyg.2012.00416

This article was submitted to Frontiers in Theoretical and Philosophical Psychology, a specialty of Frontiers in Psychology.

Copyright (C) 2012 Yordanova, Kolev and Kirov. This is an open-access article distributed under the terms of the Creative Commons Attribution License, which permits use, distribution and reproduction in other forums, provided the original authors and source are credited and subject to any copyright notices concerning any third-party graphics etc. 